\title{
SWAS Observations of Comet 9P/Tempel 1 and Deep Impact
}

\author{
Frank Bensch ${ }^{1,2}$, Gary J. Melnick ${ }^{2}$, David A. Neufeld ${ }^{3}$, \\ Martin Harwit ${ }^{4}$, Ronald L. Snell ${ }^{5}$, and Brian M. Patten ${ }^{2}$ \\ ${ }^{1}$ Radioastronomisches Institut, Universität Bonn, Auf dem Hügel 71, 53121 Bonn, Germany \\ ${ }^{2}$ Harvard-Smithsonian Center for Astrophysics, 60 Garden Street, Cambridge, MA 02138, USA \\ ${ }^{3}$ Department of Physics and Astronomy, Johns Hopkins University, Baltimore, MD, USA \\ ${ }^{4} 511$ H Street, SW, Washington, DC, USA; also Cornell University \\ ${ }^{5}$ Department of Astronomy, University of Massachusetts, Amherst, MA 01003, USA
}

\begin{abstract}
On 4 July 2005 at 5:52 UT the Deep Impact mission successfully completed its goal to hit the nucleus of $9 \mathrm{P} /$ Tempel 1 with an impactor, forming a crater on the nucleus and ejecting material into the coma of the comet (A'Hearn et al. 2005). The $370 \mathrm{~kg}$ impactor collided with the sunlit side of the nucleus with a relative velocity of $10.2 \mathrm{~km} \mathrm{~s}^{-1}$. NASA's Submillimeter Wave Astronomy Satellite (SWAS) observed the $1_{10}-1_{01}$ ortho-water ground-state rotational transition in comet $9 \mathrm{P} /$ Tempel 1 before, during, and after the impact. No excess emission from the impact was detected by SWAS. However, the water production rate of the comet showed large natural variations of more than a factor of three during the weeks before the impact.
\end{abstract}

Keywords. comets: individual (9P/Tempel 1) — radiative transfer — radio lines — solar system

SWAS is a complete space-borne radio observatory (Melnick et al. 2000), capable of observing the $556.9 \mathrm{GHz}$ transition of ortho $\mathrm{H}_{2}^{16} \mathrm{O}$, among other species, with a velocity resolution of $1 \mathrm{~km} \mathrm{~s}^{-1}$ and a beam size of $3.3 \times 4.5^{\prime}$. SWAS began near-daily monitoring observations of comet 9P/Tempel 1 on 5 June 2005, and the observations were continued post-impact until 1 September 2005. The total water production rate $Q$ of the comet is determined from the velocity-integrated intensity of the emission detected in the SWAS spectra (typically being 2- to 3-day co-adds) and employing the radiative transfer model for water line emission in comets by Bensch \& Bergin (2004). The left panel of Figure 1 shows the SWAS-measured water production rate for the observations made from 5 June through 9 July. The cometary activity traced by the water evaporation rate varies by more than a factor of three, from $3.8 \times 10^{27}$ to $12.9 \times 10^{27}$ molecules per second (115 to $385 \mathrm{~kg} \mathrm{~s}^{-1}$ ), respectively.

No statistically significant increase in the water line emission was detected by SWAS immediately following the impact. The average velocity-integrated intensity during the three days after the impact was $0.16 \pm 0.04 \mathrm{~K} \mathrm{~km} \mathrm{~s}^{-1}$, virtually identical to the average intensity measured for the three days before impact (Fig. 1, right panel). This corresponds to a total water production rate of $(6.6 \pm 1.5) \times 10^{27} \mathrm{~s}^{-1}$.

In order to derive an upper limit on the water released by the impact we extended the radiation transfer model to include comets where the water production rate is timedependent. The total water production rate is replaced by $Q=Q_{q}+Q_{b}(t)$, where $Q_{q}$ is a constant (quiescent) component. $Q_{b}(t)$ is the time-variable component, assumed to be a box-car function for the present simulations. In this case the water production rate is elevated (but constant) for the duration $\tau$ of the outburst and returns to the preoutburst level afterwards. The total number of water molecules released by the outburst is 

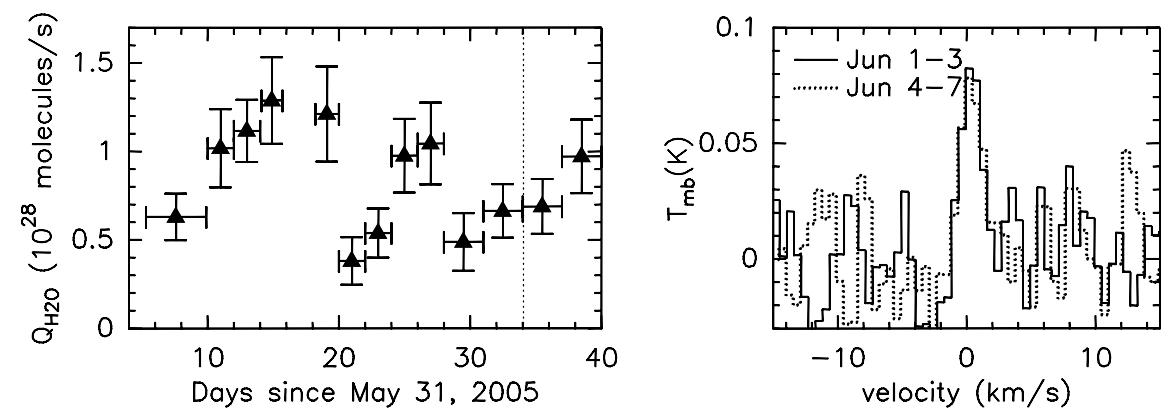

Figure 1. Left: Water production rate of comet 9P/Tempel 1, derived from SWAS observations of the $1_{10}-1_{01}$ transition of $\mathrm{o}-\mathrm{H}_{2}^{16} \mathrm{O}$. The vertical line gives the impact time. Right: SWAS spectrum before and after the impact. Each spectrum is a co-average of 3 days.
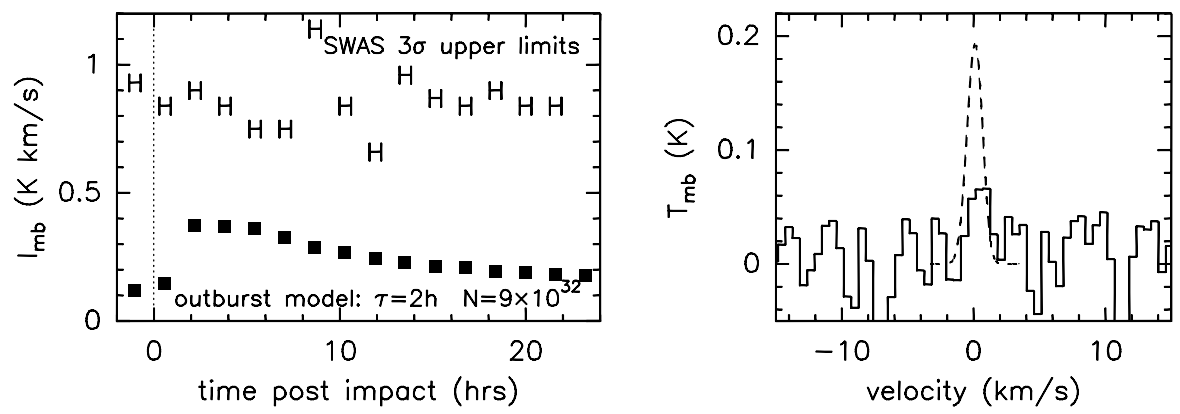

Figure 2. Left: The velocity-integrated intensity calculated by an outburst model with $\tau=2 \mathrm{hrs}$ and $N=9 \times 10^{32}$ is compared to the $3 \sigma$ upper limit derived from the SWAS spectra observed during each orbital segment of 35 min duration (error bars). No emission is detected in individual segments, consistent with the low $Q_{\mathrm{H}_{2} \mathrm{O}}$ measured before impact. Right: the weighted average of line profile for this outburst model (dashed line) is compared to the SWAS-measured spectrum. The individual spectra are weighted by the square of integrated intensity predicted by the model for each orbital segment and the inverse square of the rms noise in the spectrum.

$N=Q_{\mathrm{b}} \times \tau$. An example for the time evolution of the velocity-integrated intensity derived with this outburst model is shown in Figure 2. Preliminary model results for the SWAS observations up to $24 \mathrm{hrs}$ after the impact give a $3 \sigma$ upper limit of $\sim 9 \times 10^{32}$ molecules $\left(2.7 \times 10^{4}\right.$ tons $)$ for the vaporized water. This result is not very sensitive to the assumed duration of the outburst, as a similar upper limit is obtained for models with $\tau=0.5$, 2,8 and 16 hrs. In addition, a doubling of the water production rate can be excluded at a $3 \sigma$ confidence level if we assume that the impact created a new, permanently active area on the comet nucleus. Further results from the Deep Impact event are highlighted by D. Despois (this volume).

\section{Acknowledgements}

Support of the SWAS mission is provided by NASA through SWAS contract NAS530702. Additional support for the SWAS 9P/Tempel 1 observing campaign was obtained by the Deep Impact team. The help and cooperation of NASA HQ is acknowledged.

\section{References}

A'Hearn, M.F., et al. 2005, Science 310, 258

Bensch, F. \& Bergin, E.A. 2004, Ap. J. 615, 531

Melnick, G.J. 2000, Ap. J. 539, L77 